\title{
Caminos coloniales hacia el mundo moderno. Narrativas de la conquista de América en libros de texto alemanes y mexicanos contemporáneos
}

Colonial Pathways to the Modern World. Narratives of the Conquest of America in Contemporary German and Mexican Textbooks

\section{Daniel Schumann}

Albert-Ludwigs-Universität Freiburg, Alemania daniel.schumann@gmx.de.

\section{Resumen}

Este artículo examina desde una perspectiva comparativa la narrativización y funcionalización de la conquista de América en los libros de texto escolares actuales de Alemania y México (publicados entre 2009 y 2014). Se usa el método del análisis del discurso con enfoque en la incorporación del acontecimiento histórico en macronarrativas, se estudian también diferentes modos de establecer imaginarios hegemónicos de interculturalidad a través de la narración de la conquista. Argumento en que, a pesar de que las representaciones históricas difieran, pueden observarse silenciamientos epistémicos semejantes.

Palabras clave: libros de texto, análisis del discurso, historiografía, colonialismo, interculturalidad.

\begin{abstract}
This article examines the narrativization and functionalization of the Conquest of America in current German and Mexican school textbooks (published between 2009 and 2014) from a comparative point of view. Using a discourse-analytical approach and focusing on the incorporation of the historical event into macro-narratives, different modes of establishing hegemonic imaginaries about interculturalism through the narration of the Conquest are studied. I argue that in spite of the differences in historical representations, similar epistemic silencings can be observed.
\end{abstract}

Abstract

Keywords: Textbooks, Discourse analysis, Historiography, Colonialism, Interculturalism. 


\section{Introducción}

La conquista de América es, sin duda, uno de los acontecimientos históricos más importantes de la historia mundial, y es considerada por varios científicos sociales e historiadores como el inicio de la era moderna, del colonialismo y de la globalización. En su famoso estudio La conquista de América. El problema del otro, el sociólogo Tzvetan Todorov analiza el acontecimiento histórico en su "valor paradigmático" de contacto cultural y, al examinar sus consecuencias para la actualidad, concluye que "el descubrimiento de América es lo que anuncia y funda nuestra identidad del presente" (Todorov, 1998: 15). La conquista también juega un rol central para el filósofo Enrique Dussel, quien interpreta el año 1492 como el "nacimiento de la modernidad", a partir del cual Europa se constituye como centro del mundo, mientras que al mismo tiempo América pasa a ser "su otra cara, su lado explotado, dominado y cubierto" (Dussel, 1993: 15, 62). Tanto Dussel como Todorov -a pesar de sus diferenciasconcuerdan en la doble fractura que atribuyen al evento: como cierre geográfico del mundo, por un lado, y como fuerte discontinuidad temporal, por el otro. Es esta doble fractura la que hace que hasta hoy día la conquista sea un importante punto de partida para varias macronarrativas y políticas identitarias; sin embargo, en su integración en las macronarrativas que son relevantes para las sociedades actuales, la construcción histórica del hecho de la conquista es reñida y se encuentra en un constante proceso de negociación social, dependiente de los discursos y relaciones de poder presentes. El propósito de este estudio es analizar la construcción histórica de la conquista de América en los libros escolares de Alemania y México publicados entre 2009 y 2014, para mostrar así diferentes modos de narrativizar los inicios de la historia del colonialismo moderno mediante marcos interpretativos coloniales a los cuales los medios educativos recurren.

A través de un análisis del discurso comparativo enfocado a las narraciones de los libros escolares y sobre la base de teorías historiográficas y decoloniales, en este artículo estudio la representación y funcionalización del inicio del colonialismo en los libros de texto de historia de México y Alemania y sus implicaciones para el presente. Una comparación de materiales educativos de estos dos países es particularmente interesante, ya que se contrastan los libros de texto de un país anteriormente colonizado, con una relación espacial directa con la conquista, con los libros de un país de una larga historia colonial que, tanto en la conciencia pública como en su discurso científico, pasó y pasa ser en gran medida ignorada (Eckert y Wirz, 2002). Se desarrolla la hipótesis de que los hechos históricos construidos en los medios educativos analizados no sólo trasmiten conocimientos sobre la conquista de América, sino también a través de ella; es decir, más allá de narrar una serie de eventos históricos, los libros de texto proponen consecuencias más generales, y construyen así imaginarios sobre culturalidad e interculturalidad para las respectivas sociedades contemporáneas. Después de 
presentar el marco teórico y metodológico del estudio reconstruiré los ejes centrales de las narraciones sobre la conquista para mostrar que, en los dos casos, la conquista de América sirve para establecer dos macronarrativas: la dominación europea en el caso de los libros de texto alemanes, y la hibridación en el caso mexicano. Termino discutiendo, con herramientas de teorías poscoloniales, si los libros de texto de ambos países logran "llegar desde una representación colonial del mundo hacia una representación del mundo colonial" (Macgilchrist y Otto, 2014: 4).

\section{Lecciones históricas: la construcción del pasado desde el presente}

Parto del presupuesto teórico de que el pasado no es algo objetivamente dado, que puede conocerse "tal y como realmente fue" (según la famosa expresión de Leopold von Ranke). A diferencia de una representación supuestamente objetiva del pasado, las teorías historiográficas neohistoricistas -sigo aquí a Jürgen Pieters (2000: 21ss.) - entienden el pasado como una polifonía de voces y contravoces, lo que conduce a que la historia no sea algo monolítico. El neohistoricismo argumenta que el o la investigadora tiene su propia historicidad y que escribe la historia desde un posicionamiento específico, siempre relacionado con el episteme de una cierta época y un lugar. Además, esta corriente critica la teleología muchas veces inherente en las representaciones del pasado que atribuye a los acontecimientos históricos una finalidad. En coincidencia con ese enfoque, en este trabajo comprendo a la historia analíticamente como producto de una práctica que tiene lugar en el presente con una lógica específica. En este sentido, me interesa observar cómo la historia está "hecha" (Certeau, 1991: 31), es decir, cómo los conocimientos históricos son producidos a través de prácticas historiográficas presentes que siempre forman parte de procesos políticos en un sentido amplio. Sin embargo, mientras que los trabajos del neohistoricismo se centran mayormente en las prácticas científicas de la historiografía, en este artículo intento usar sus herramientas para analizar prácticas historiográficas de medios educativos como libros de texto escolares. A continuación se expone el marco teórico de la investigación en diálogo con las teorías del neohistoricismo, pero se toma en cuenta también la lógica específica de los medios educativos y su forma de producir conocimiento histórico.

Si la historia es el "resultado de una práctica actual" (Certeau, 1991: 86), es importante analizar sus métodos, contextos y condiciones de producción. Según Certeau (1991: 11ss.), la operación historiográfica funciona a través de la diferencia principal entre el presente y el pasado, siendo el pasado un objeto en una lengua descifrable que los historiadores tratan de entender y "hacer hablar". Este proceso de escribir la historia puede ser caracterizado por dos 
procesos simultáneos: primero, cada tipo de historiografía está basada necesariamente en una reducción de complejidad y una selección entre los acontecimientos que se pueden y quieren narrar y los que no son considerados relevantes para ocupar un lugar en la historia. En este sentido, la selección de acontecimientos históricos es "voluntarista" y depende del sujeto enunciador y de los contextos y condiciones de producción en las cuales se encuentra (Certeau, 1991: 14); segundo, cada selección está siempre ligada a una "semantización" (Certeau, 1991: 121) o a una narrativización, ya que un acontecimiento no sólo se selecciona así "como realmente fue", sino que, para lograr una facticidad, siempre se trata de un "evento bajo descripción", como lo denomina el filósofo e historiador Hayden White (2008: 13). La historia, vista así, es el producto de una transformación de eventos pasados -que la historiografía trata de alcanzar, pero que siempre tendrá un punto ciego- a hechos históricos a través de la narrativización; en otras palabras, se trata de separar un evento de su contexto original, a la vez que se lo describe y reconfigura para otorgarle facticidad. Sin embargo, es obvio que un acontecimiento no puede ser narrado aisladamente, sino siempre en relación con otros acontecimientos y procesos históricos. La pregunta que plantea este artículo es, precisamente, cómo el "macrohecho" de la conquista de América está producido narrativamente, y se nutre de una reconfiguración de eventos históricos que se relacionan con macronarrativas más abstractas y, en parte, teleológicas.

Desde la perspectiva neohistoricista, la escritura de la historia se perfila entonces como un proceso político de producción de factualidad y coherencia histórica a través de selección y semantización. Los hechos históricos, según White, son observaciones de segundo orden: "[T]hey are events in speech about other speech events and other kinds of events beyond or outside of speech" (White, 2008: 13). Por consiguiente, la historia, "tal y como realmente fue", siempre es el resultado de un proceso comunicativo desde un lugar actual específico y, por ende, entrelazado con intereses, demandas y relaciones de poder de la sociedad contemporánea. Desde una perspectiva sociológica, la historia puede considerarse funcional para el presente, ya que, por ejemplo, se usa el pasado como área de proyección para dar continuidad a un grupo específico a través de la "invención de la tradición" (Hobsbawm, 1984) o, por otro lado, se usa el pasado como demarcación y entidad que debe ser superada. La historiografía, sea la científica o la educativa, tiene un papel performativo para el presente si es considerada "una práctica social que asigna un lugar específico a sus lectores, ya que redistribuye el espacio de referentes simbólicos y les da una lección" (Certeau, 1991: 114). Es por esto que en el presente artículo no me limito a analizar solamente la producción de conocimiento sobre el pasado, sino que examino la producción de conocimiento a través del pasado y su performatividad para el presente. En este punto es importante reconocer el mérito de los estudios poscoloniales y decoloniales que han investigado críticamente por quién y desde 
dónde están dadas estas lecciones (Chakrabarty, 1992; Escobar, 2003), por lo cual forman parte imprescindible del marco teórico aquí empleado.

Ahora bien, si el análisis de la operación historiográfica de Michel de Certeau y Hayden White es una herramienta adecuada para investigaciones sobre todo tipo de producción de conocimiento histórico, es importante aún mencionar las especificidades y contextos del medio educativo y su forma de historiografía. La manera de narrar la historia en libros de texto puede considerarse, por un lado, como una derivación tardía del discurso científico. Los medios educativos retoman los resultados de investigaciones históricas con retraso, lo cual conduce a que el conocimiento histórico representado en los libros de texto muchas veces difiera de la discusión científica actual (Bernhard, 2013: 207; Höhne, 2003: 158). Respecto del tema de la conquista de América en los libros de texto, un extenso estudio del historiador Roland Bernhard (2013) muestra bien esta diferencia: Bernhard compara la narración de la conquista en libros de texto alemanes y austriacos con el discurso actual científico sobre el tema. Mientras que el discurso de la ciencia histórica se centra en los intercambios transculturales, los grupos de actores interconectados y el intento de diferenciar el fenómeno del coIonialismo, los libros de texto en lengua alemana, según Bernhard, dan una imagen bastante diferente de la situación, ya que son el resultado de categorizaciones totalizantes y atribuciones de agencia unilateral. Así, por ejemplo, la colonización de América se describe a menudo en los libros escolares alemanes como un proyecto llevado a cabo por españoles "activos" contra indígenas representados como pasivos. Cabe mencionar que este aspecto es estudiado en la investigación actual de manera más diferenciada y con interés en los actores "conquistados", cuya agencia abarca desde la cooperación hasta la resistencia (Osterhammel y Jansen, 2012).

Por otro lado, más allá de su relación con el discurso científico-histórico, los libros de texto deben considerarse como un medio específico y complejo de la producción de conocimiento, hecho ampliamente mostrado por la investigación manualística. ${ }^{1}$ Los libros de texto producen discursos relativamente oficiales y hegemónicos, ya que de alguna u otra manera son controlados por el Estado (Stöber, 2010; Anzures, 2011) y reflejan el resultado de un largo proceso de influencias y negociaciones sociales. Desde la perspectiva de la teoría del discurso, un libro de texto es un vehículo de mediación (Höhne, 2003; Heinze, 2014): por una parte, es el producto de la negociación de otros discursos sociales; por la otra, es un productor de discursos que reconfigura y construye conocimientos según su lógica medial

\footnotetext{
${ }^{1}$ Véase para una buena introducción a la investigación manualística y sus metodologías y métodos la compilación de Petr Knecht et al. (2014) y, desde la perspectiva francesa, un texto clásico de Alain Choppin (2001) con enfoque histórico que brevemente define el medio del manual escolar. Para la investigación manualística en Alemania véase el Georg-Eckert Institut für internationale Schulbuchforschung (CEI; Instituto Georg Eckert para la Investigación Internacional sobre Libros de Texto).
} 
específica. Es un medio que debido a la trasmisión selectiva de conocimiento no sólo es restrictivo, sino también es productivo en la medida en que hace pedagógicamente accesibles diversos temas y que predetermina la manera de tematizarlos en los salones. Al trasmitir entonces conocimiento controlado tanto como "controlante", los libros de texto de historia no sólo construyen sus objetos en relación con las premisas de la historiografía hegemónica, sino también respecto de las normatividades pedagógicas y de la política educativa. Estos requerimientos normativos pedagógicos influyen en la toma de decisiones acerca de los temas que serán incluidos en el libro de texto y cuál será su finalidad didáctica. Un concepto cada vez más importante en Alemania y en México que en los últimos años ha cambiado los currículos y los libros de texto es el discurso de la educación basada en competencias (Keller, 2010; Díaz Barriga, 2006; Neyra Galicia, 2010). Los currículos actualmente se centran más en enseñar disposiciones que posibilitan una "conducción de sí flexible" (Keller, 2010). Por esto puede asumirse que la construcción del objeto de la conquista en los libros de texto de historia no sólo depende de la selección y construcción de los meros hechos históricos, también se orienta según la posibilidad de trasmitir y propiciar otras disposiciones básicas a través de la conquista, que van más allá del tema histórico. En el caso de la conquista, los currículos alemanes y mexicanos mencionan, respecto del significado del tema para el presente, las competencias interculturales (SEP, 2011, 2013; Hessisches Kultusministerium, 2011). Es justamente la producción de este conocimiento normativo para el contacto intercultural de hoy a través de la construcción del pasado, el tema central del estudio de caso que será presentado a continuación.

\section{Metodología del estudio de caso²}

Para el estudio de caso se seleccionaron libros de texto recientes de historia de Alemania y México. En una carrera educativa, el tema del "descubrimiento" y la conquista de América aparece varias veces, así que era importante reducir el corpus de manera sistemática. En el caso alemán, pese a su política educativa federal, se seleccionaron los libros de texto del estado de Hessen, publicados entre 2012 y 2014, como pars pro toto. En el nivel de secundaria se incluyó un libro de cada tipo de colegio - de Haupt und Realschule (ZR), Realschule (Ev) y Gymnasium (Hz). Además, se incluyó un libro de texto (KG) del nivel de Gymnasiale Oberstufe, los últimos años de la formación escolar en Alemania. En el caso mexicano, la selección fue más difícil, ya que el tema aparece en un espectro más amplio. En el nivel de primaria se incluyeron en el análisis los libros de texto gratuitos oficiales de cuarto y sexto

\footnotetext{
2 Para un mejor flujo, se usan abreviaturas para citar los libros de texto analizados. Los libros de texto correspondientes a las siglas aquí empleadas se encuentran en la bibliografía.
} 
grado, publicados en 2010 (PR4) y 2009 (PR6), mientras que no se pudieron tomar en cuenta los libros de texto gratuitos regionales, en los cuales la conquista de México también está tematizada en algunos casos. Para secundaria, se incluyeron dos libros de texto de editoriales privadas; cada uno representa un año en el cual el acontecimiento está tematizado según el currículo (H2; HU). Con la selección de estos materiales no se pretende dar una visión completa sobre cada tipo de representación de la conquista de América en libros de texto mexicanos y alemanes, sino más bien una base desde la cual se pueden explorar y analizar los mecanismos narrativos de presentación del evento histórico y el sentido que se le da para el presente. El mecanismo analítico central del estudio es la mirada comparativa; es decir, la lectura de los libros de texto alemanes desde la perspectiva de los mexicanos y viceversa, con lo que se pretende dar a conocer con más claridad las especificidades narrativas de los dos corpus tanto como las ausencias de cada forma de narrar la historia. ${ }^{3} \mathrm{El}$ análisis del discurso con enfoque en lo narrativo, basado en parte en el método de Willy Viehöver (2006), se centró en cuatro planos: 1) la construcción del acontecimiento histórico de la conquista de América y las maneras de situarlo en el contexto histórico; 2) las omisiones y las ausencias en la narración con base en una comparación de los dos corpus; 3 ) la funcionalización de la conquista y las lecciones para el presente, y 4) la manera de revelar -o no revelar- la contingencia de la narración y, por consiguiente, de dar lugar a una autocrítica.

A continuación se exponen algunos resultados del análisis de la narración alemana y mexicana de la conquista de América. Primeramente se da una visión de conjunto de la construcción del objeto histórico en los respectivos manuales escolares, para después examinar los mecanismos narrativos, enfocados específicamente en la relación de la presentación del evento histórico de la conquista con otras macronarrativas relevantes para los imaginarios actuales de interculturalidad.

\section{El caso alemán: construir el dominio europeo El contexto narrativo: nuevos tiempos, nuevos mundos}

Todos los libros de texto alemanes aquí analizados tratan la conquista de manera parecida. Lo que está presentado es la historia de profundos cambios en Europa alrededor del año

\footnotetext{
${ }^{3}$ Me centro aquí en una comparación sincrónica. Sin embargo, una investigación genealógica de la representación y funcionalización de la conquista en los libros de texto sería igualmente importante. Ya existen estudios que serían un buen punto de partida para una comparación diacrónica; por ejemplo, el estudio de Lamoneda Huerta (2004), que analiza la enseñanza del descubrimiento y de la conquista de América en los libros de texto mexicanos publicados entre 1992 y 1994. Además, véase una compilación coordinado por Pérez Siller (1992) que examina la representación de la conquista a través de los libros de texto de países de la misma época.
} 
1500. Los capítulos relevantes sugieren que se trata de una época que representa lo nuevo per se, con títulos como "Partida hacia un tiempo nuevo" (Hz), "Nuevos horizontes-nuevos mundos" (EV) o "Caminos hacia el mundo moderno" (KG). Por lo tanto, en el caso alemán las representaciones de la conquista y de la primera fase del colonialismo sólo pueden analizarse en su incorporación en dicha narrativa del "tiempo nuevo". En los libros de texto, los acontecimientos del "descubrimiento", la conquista y la colonización de América se exponen después de la descripción de los "mundos de vida medievales" (HZ), la Reforma, los cambios religiosos y el absolutismo europeo. De ahí, en los capítulos sobre la edad moderna la historia centrada en Europa hace un breve excurso a su "exterior", pero regresa pronto -como Cristóbal Colón- a su enfoque eurocéntrico. A continuación se esbozan los pasos que hace la narración de los libros de texto de los cambios en el umbral de la edad moderna que finalmente llevan a una descripción de la conquista enmarcada en una narrativa del surgimiento de Europa.

En una primera etapa todos los libros de texto alemanes narran la historia de una transición radical de un orden medieval, fruto de un designio divino, hacia una época con nuevos signos. Mientras en la Edad Media todos tenían su lugar fijo, ahora nace una nueva época con el "hombre en el centro" (ZR: 68) que se vuelve "la medida para todo" (HZ: 126). Los libros de texto presentan la formación de una "nueva mentalidad" ( $\mathrm{HZ}$ : 126) del Renacimiento y del humanismo, con una sociedad conformada por ciudadanos "autoconscientes, orgullosos y felices" que quieren superar los márgenes medievales: "Por eso, era necesario observar propiamente, coleccionar, comparar y llegar a sus propias conclusiones" (EV: 108). Los libros de texto declaran el nacimiento de una nueva cientificidad que parece ser el motor más importante de un proceso de liberación mental europea. Pero el nuevo concepto del hombre no solamente libera fuerzas mentales, sino también se manifiesta en nuevos descubrimientos: "Las invenciones cambian la vida" (ZR: 70); entre ellas, la imprenta y la transición del modelo geocéntrico al modelo heliocéntrico, pero también la invención de la pólvora, de nuevos instrumentos de navegación y tipos de naves, entre otros. Todos los capítulos alemanes introducen dos personajes como tipos ideales de la época de invenciones, el "hombre universal" Leonardo da Vinci (KG: 147) y Galileo Galilei. En la narración, los dos comparten el mismo espíritu científico y representan la ruptura valiente y heroica con el orden medieval; a la vez que comparten también un antagonista común que siempre "quería restringir su investigación" (ZR: 70): la Iglesia. En la narración, la Iglesia es concebida como un obstáculo para la nueva época, el cual, sin embargo, es superable mediante la valentía y el empirismo moderno. Sólo un libro de texto indica, en cambio, la coexistencia de la Iglesia con la nueva mentalidad, ya que "no era un rechazo de la creencia cristiana", sino que se trataba más bien de "una liberación de la Iglesia y el cristianismo de las 'aberraciones' de la Edad Media" (KC: 147). En la narración hegemónica de los libros de texto alemanes el nuevo espíritu científico 
y valiente europeo junto con sus efectos a través de las invenciones son la quintaesencia que sirve de trasfondo para la presentación de los acontecimientos del inicio del colonialismo.

A continuación, los libros de texto introducen un subcapítulo que trata el "descubrimiento" de América. El punto de partida es un evento histórico que impide el desarrollo del actor colectivo Europa, el cual, según los libros de texto, ya se había constituido hasta entonces: "En 1453, los turcos musulmanes conquistaron Constantinopla. [...] Para mercancías, tomaron una aduana alta, así que subieron los precios en Europa" (ZR: 76). A partir de este evento, en la narración de los libros de texto ya no son sólo los científicos, sino todo el actor colectivo de Europa el que está siendo bloqueado por su "exterior" geográfico-cultural. La toma de Constantinopla es el motivo inmediato para la búsqueda de nuevas rutas por los portugueses y los españoles, lo cual está mencionado en todos los capítulos alemanes. En este sentido se menciona a Cristóbal Colón que, gracias a las "invenciones técnicas" (EV: 114) y los "nuevos procedimientos científicos" (HZ: 139), por un lado, e impulsado por el "nuevo concepto del humano del renacimiento" - "valentía y confianza en las propias capacidades" (HZ: 138) - por el otro, inicia su "partida hacia lo desconocido" (EV: 116). Llama la atención que la llegada de Colón y el contacto cultural con los habitantes de la isla de Guanahaní tenga relativamente poca importancia en la narración misma. El momento del denominado "descubrimiento" está excluido del flujo del texto -igual que la muy escasa reflexión del término-y se ve desplazado a las instrucciones de trabajo en el plano didáctico de los libros de texto.

De ahí, los libros de texto (menos KG) tratan la conquista de México, y sólo aquí se tematiza que el "Nuevo Mundo" no es tan nuevo, ya que "en este continente hace mucho tiempo vivían numerosos pueblos con alta cultura como los mayas, los incas y los aztecas" (EV: 118). Si bien para la descripción del "descubrimiento" la narración se centra más en la geografía y los productos, en el caso de la conquista los habitantes y las culturas del territorio mexicano representan la contraparte de los conquistadores españoles, aunque su presencia, como se verá, sea de corta duración. Comenzando con la información de que los españoles se encontraron con los aztecas, los libros de texto alemanes introducen la cultura, la vida cotidiana y las estructuras sociales de estos últimos. Comparten el mismo modo de descripción cuando clasifican a su cultura como muy avanzada, caracterizada por "capacidades excelentes de técnica y organización" (Hz: 142), hecho que, como los libros no se cansan de subrayar, causa asombro entre los españoles; sin embargo, en gran medida los capítulos alemanes representan a los aztecas como una cultura estática: en su excurso fuera de los espacios culturales europeos, la narrativa europea no entra en contacto con diferentes sociedades dinámicas y espacios políticos reñidos, sino con una cultura fija con casi ninguna historicidad e interacción. La cultura de los aztecas sólo se vuelve relativamente dinámica con el inicio de la guerra, "con cruz y espada" (ZR: 88), por los españoles; no obstante, se 
destaca claramente la supuesta pasividad de Moctezuma. Aunque algunos libros de textos hacen notar las resistencias de los aztecas, la historia de la conquista está mayormente representada como un proyecto realizado únicamente por Hernán Cortés y sus seguidores españoles.

Para el recuento de la época posterior de la conquista, la narración entra en un subcapítulo que describe el inicio del dominio colonial y la explotación, y sondea las consecuencias fatales para los conquistados y los esclavos. Se centra en el "comercio con ganancias" (EV: 127) para Europa tanto como en su cara oscura. En una conclusión más abstracta, todos los capítulos vuelven a la Europa empoderada que describieran al inicio de la narración, para localizar las consecuencias de la conquista dentro de una narrativa de "la 'europeización' del mundo" (HZ: 143). Aquella Europa que se ha constituido como la cuna de un espíritu nuevo se vuelve el actor central del dominio global, lo que en las colonias "tiene consecuencias negativas hasta hoy" ( $\mathrm{Hz}$ : 143), como reza el resumen de los libros de texto. A pesar de todo, mientras que se toma en cuenta la colonización con sus consecuencias nefastas para las colonias, la evaluación de los efectos culturales del colonialismo para la propia Europa está prácticamente ausente. Según la narrativa aquí reconstruida, el lado oscuro de la europeización no parece llegar a las costas de la misma Europa.

\section{La superioridad cultural europea como condición de la conquista}

El resumen expuesto demuestra la incorporación inseparable del evento de la conquista en una macronarrativa de la constitución y la universalización de la cultura europea. La historia contada en los capítulos alemanes es la del inicio de la era moderna: "Comenzó la edad moderna, la época a la cual también pertenece nuestro presente" (ZR: 65). Mi argumento es que los libros de texto alemanes proponen una lectura de la historia en la cual Europa se constituyó intrínsecamente como la cultura superior antes del contacto con el denominado "Nuevo Mundo". Aquella "dinámica civilizatoria que proporcionó al continente una superioridad ante otras culturas" (KG: 148), su intrepidez y su cientificidad son mencionadas como requisitos de la conquista; la conquista, en cambio, es concebida implícitamente como el cumplimiento de la fuerza cultural europea desarrollada por sí misma. Por consiguiente, la narrativa alemana está diametralmente opuesta al análisis del filósofo argentino Enrique Dussel (1993), quien argumenta que dicho actor colectivo europeo sólo pudo constituirse como centro superior del mundo a través de la conquista y la consecutiva demarcación de su "otro colonial" americano. En este sentido, la conquista no es el efecto de la superioridad europea, como sugieren los capítulos aquí analizados, sino más bien su condición. 
Los libros de texto construyen esta superioridad a través de una reconfiguración de la cronología del flujo de eventos históricos en una línea nueva. Se trata de una ruptura con la cronología "real" - un tipo ideal de la historiografía - para establecer su propia narración lineal y didáctica de la historia. En este caso, es interesante que eventos e invenciones de un lapso de cuatro siglos estén condensados en un solo punto de la narración para representar el espíritu nuevo de Europa. La invención del reloj mecánico (1300), la imprenta (1458), la publicación de Copérnico sobre el modelo heliocéntrico en 1543 y, 90 años más tarde, la disputa de Galileo con la Iglesia: todos estos eventos están narrativamente condensados y situados antes del contacto cultural con América y explícita e implícitamente son tomados como condiciones de la conquista:4 "Difícilmente pueden imaginarse los viajes de exploración sin el concepto humano del renacimiento" y la nueva cientificidad (HZ: 138s.). Para los libros de texto, por el contario, el contacto con América tiene poca relevancia cuando se trata de la consolidación de la superioridad cultural europea.

Con la reconfiguración cronológica se entremezcla un segundo mecanismo narrativo que sugiere una concepción específica del contacto cultural que involucra a los europeos. El actor colectivo de los europeos, que sin mayor diferenciación constituye la base implícita de la narración, figura como un monolito. Según los libros de texto alemanes, Europa aparece como un actor unificado que sólo tiene logros y finalidades comunes, mientras que no se especifican ambivalencias o diferencias entre los actores europeos. Aunque varios actores organizaban viajes de exploración, con mandatos distintos, Europa está representada en su convivencia armónica para llevar a cabo la "europeización del mundo"; sólo posteriormente "los europeos peleaban por sus colonias" (ZR: 92). Un aspecto que también evoca esta impresión monolítica es que en muy pocos casos la narración considera intercambios culturales entre Europa y actores de otras regiones en la producción de conocimiento que llevaría a la superioridad cultural europea. Si bien Europa puede ser bloqueada y desafiada por su "afuera cultural", en la narración hegemónica no parece recibir algo de sus aportes. Europa supuestamente logró su desarrollo y superioridad cultural intrínsecamente y antes de la conquista; sólo después, en la época colonial, se ve su cara oscura.

\section{Redescubriendo el "descubrimiento"}

Aparte de la vinculación con la macronarrativa de la superioridad europea, la narración propia del "descubrimiento" y la conquista puede caracterizarse por una perspectiva específica de redescubrir la historia. La narración hegemónica de los libros de texto alemanes no sola-

\footnotetext{
${ }^{4}$ Es llamativo que la Reconquista no es mencionada como condición relevante. Para una discusión del nexo entre la Reconquista y la conquista de América véase por ejemplo Böttcher 2013.
} 
mente sigue los pasos de los descubridores, sino también sugiere repensar sus pensamientos, ver a través de sus ojos y contemplar el esplendor del mercado de Tenochtitlán, de la misma manera cómo lo hizo Hernán Cortés casi 500 años atrás. Si América fue "descubierta" en 1492, los libros de texto alemanes aquí analizados en gran parte no hacen otra cosa que redescubrir el continente a través del lente conquistador.

La perspectiva del descubrimiento repetido se muestra primero en el ámbito epistemológico de la narración. ¿Cómo se entera el lector de los libros de texto acerca de las sociedades no europeas y sus historias? La conquista no es narrada desde una perspectiva multicéntrica — con sociedades diferentes e historias distintas - sino desde una perspectiva eurocéntrica. Para aprender algo de América hay que repensar los pensamientos estratégicos de Colón ("También puedo llegar a India si navego hacia el oeste"; ZR: 80), reconstruir la técnica de sus barcos y, al final, abordar uno de ellos. Lo mismo puede observarse en la narración de la conquista de México por Hernán Cortés: Sobre la cultura azteca sólo se aprende en el mismo momento en el que se exponen los planes de conquistarla. El breve excurso a otra cultura - más breve o ausente es el excurso a otra historia - es completamente dependiente de la perspectiva estratégica de los conquistadores españoles; epistemológicamente se aclara sólo lo que ella permite y cuando lo permite. Por otra parte, una convergencia de la perspectiva de la narración del libro de texto y la perspectiva de los conquistadores se muestra en el aspecto de la valoración. Una comparación entre la narración dominante de los libros de texto y el famoso análisis hermenéutico de Tzvetan Todorov (1998) de los diarios de Colón y Cortés aclara paralelos: la connotación en la narración es positiva cuando Cortés está fascinado (por el mercado o las competencias técnicas de los aztecas, por ejemplo) y se evidencia un distanciamiento cuando él condena algo (en primer lugar la práctica de los sacrificios humanos).

En el ámbito semántico-conceptual, la presentación de los acontecimientos históricos funciona en gran parte a través de un vocabulario que fue usado por los propios conquistadores o que se desarrolló en la época colonial. Cuando en los libros de texto los españoles entran en contacto con los habitantes de América, si no son los aztecas o representantes de otras "altas culturas" se les denomina "indios" (EV: 115; ZR: 90; KG: 153) —también al hablar de la población indígena actual- o "Ureinwohner" (HZ: 139; KG: 153), una categoría de la cual los habitantes del continente nunca podrán liberarse en la narración. El uso del concepto "descubrimiento" es posiblemente el ejemplo más obvio que indica el uso de un imaginario colonial que tiene una larga tradición de opresión racial. A pesar de la inclusión de fuentes y reflexiones adicionales, el texto escolar funge como un marco narrativo que conecta todos los demás elementos narrativos anteriormente expuestos. De esta manera, el texto posee la característica de implícitamente redescubrir el "descubrimiento" de los españoles. Sólo a 
través de fuentes y tareas complementarias se sugiere una reflexión didáctica, la cual sin embargo falla en mostrar la contingencia y colonialidad de su perspectiva.

\section{El caso mexicano: la genealogía de una fusión de culturas El contexto narrativo: entre lo nacional y lo global}

Comparados con los libros de texto alemanes, en los mexicanos la conquista no sólo se tematiza con más frecuencia, sino también con diferentes enfoques dependiendo del grado escolar. La colonización de América no forma parte de un solo capítulo como en el caso alemán, sino que es narrada dentro de un marco más amplio. Para dar una visión en conjunto, veremos a continuación las dos maneras en las cuales los acontecimientos relevantes están contextualizados en la narración mexicana y después revisaremos la construcción específica del "descubrimiento" de América y la conquista de México.

Entre los libros de texto mexicanos aquí analizados puede diferenciarse entre una narración con enfoque en la historia nacional —como es el caso en cuarto grado de primaria (PR4) y segundo año de secundaria (H2) - y una narración con enfoque en la historia universal en sexto grado de primaria (PR6) y primer año de secundaria (HU). La narración del enfoque nacional comienza por mencionar la primera población del continente americano, para después tematizar la "prehistoria" y las características de las culturas preclásicas mesoamericanas. Los capítulos analizados relatan la historia del ascenso de un pueblo azteca inicialmente "pobre": "tuvieron que conformarse con el lugar que les dejaron" (PR4: 28). A continuación, se cuenta cómo los aztecas pactaron con los vecinos de Texcoco y Tlacopan en la "Triple Alianza", que pronto se convirtió en "la mayor fuerza militar de Mesoamérica" (H2: 25) y que "dominó gran parte de Mesoamérica" (PR4: 30), incluso con altos tributos. Según la narración, los aztecas llegaron "de la miseria al esplendor" (PR4: 32). También se mencionan señoríos que "mantuvieron su autonomía y no se incorporaron a ninguna de las dos grandes alianzas" (H2: 25). Según los libros de texto mexicanos, a su llegada a la tierra mexicana los españoles no se encontraron con "un solo poder imperial unificado" - "si hubiera sido así, la conquista habría resultado muy difícil"-; más bien se enfrentaron con grupos que "se sentían oprimidos por las exigencias tributarias de los mexicas y sus aliados" (H2: 25). A diferencia de la narración alemana, el espacio político de Mesoamérica aquí es más dinámico y está atravesado por distintos intereses. La cultura mexica o azteca, claramente la cultura hegemónica de la narración y la que está dotada de una connotación positiva, espera la confrontación con su destino inevitable: la conquista que la transformaría 
radicalmente (H2: 13). En la exposición de las culturas "prehispánicas", la conquista es el acompañante siempre presente en el trasfondo, aviso de la transformación cultural que vendrá con los conquistadores. A partir de la llegada de estos últimos, la narración mexicana se dedica al virreinato.

La narración histórica con enfoque universal se acerca a la colonización por otras vías: En ella no se describe el final del esplendor azteca, sino el comienzo de un tiempo nuevo a través de la unión de dos historias que se convertirán en una sola historia. En secuencias alternadas se narran acontecimientos de la Europa antigua y medieval y, por otra parte, de las "culturas prehispánicas americanas". El libro de texto de primaria culmina relatando la "coincidencia con un territorio imprevisto: América" y "los inicios de la época moderna" (PR6). En esta contextualización, el "descubrimiento" como la "primera expresión del mundo globalizado" (HU: 44) también significa la primera expresión de una historia compartida: lo que se había desarrollado antes de manera separada ahora se vuelve una sola historia, pero con silencios epistemológicos que el presente artículo abordará más adelante.

¿Cómo narran los libros de texto mexicanos el acontecimiento del "descubrimiento" de América? Como en el caso alemán, los libros mexicanos no se detienen tanto en el momento de la llegada de Colón y el contacto cultural entre los españoles y los habitantes de Guanahaní; más importancia tiene la explicación de los motivos y las consecuencias de los viajes de Colón. Los motivos de los españoles, según el material analizado, son una mezcla de "la urgencia de convertir al cristianismo a los infieles, el espíritu de aventura y el deseo de conseguir riquezas" (PR4: 48). Las consecuencias, por otro lado, se perfilan como más relevantes que la motivación de la conquista y se refieren a distintos actores y esferas. Primero, una consecuencia mencionada es que los europeos "vivieron una época de expansión" (PR6: 143) y ampliaron su poder a través de la explotación económica. En segundo lugar mencionan la destrucción violenta de las culturas originarias; "el encuentro fue terrible para los habitantes del Nuevo Mundo", "la población de las Antillas casi desapareció" (PR4: 49s.). En parte, los libros de texto también enfocan la "necesidad" del tráfico de esclavos para los españoles y sus efectos. Tercero - que es para todos los libros mexicanos la consecuencia dominante-, "entrarían en contacto sociedades distintas y las influencias mutuas cambiarían sus formas de vida" (PR4: 49); "Hubo un gran intercambio de personas, conocimientos y arte, así como de flora y fauna, lo que cambió la vida de la humanidad" (PR6: 143). Esta narrativa hegemónica del enriquecimiento global y del intercambio mutuo se encuentra en una relación ciertamente conflictiva con la consecuencia de la destrucción violenta de las culturas originarias.

La misma tensión puede observarse al leer los subcapítulos de los libros de historia nacional (PR4 y H2) que se refieren a la conquista. La narración comienza con las expediciones españolas en el tiempo posterior al "descubrimiento" por Colón; la "expedición decisiva", 
en cambio, comienza en 1519, con Hernán Cortés y sus 550 (H2) o 700 seguidores (PR4), que tomaron rumbo a México, contra la voluntad del gobernador Velásquez. De ahí, la narración sigue el camino estratégico de Cortés hacia Tenochtitlán, quien conquistaría la capital azteca en los siguientes dos años. Si se compara esta representación de Cortés con la alemana, la versión mexicana del conquistador no parece de ningún modo menos poderosa y decidida; pero al seguir la epistemología del Cortés mexicano, el lector se entera de una historia distinta: en los capítulos mexicanos, la conquista no es exitosa por la fuerza brutal de Cortés en sí, sino por las interacciones estratégicas de Cortés con diferentes actores del territorio mexicano, los cuales son vistos como la verdadera causa de su superioridad. Por consiguiente, los libros de texto mexicanos presentan el acontecimiento de la conquista de manera completamente diferente: los grupos indígenas están cualitativamente integrados en la narración, ya que tienen agencia, expectativas e intenciones propias. También cambia la evaluación cuantitativa de la conquista, pues la tropa de Cortés, según los libros mexicanos, cuenta con "más de 800 españoles y cerca de 75000 guerreros indígenas" (H2: 30). Generalmente, en la narración de la conquista los actores y las relaciones entre ellos están representados de manera más diferenciada que en los libros de texto alemanes. La conquista — según la lección de los capítulos mexicanos estudiados - no hubiera funcionado sin los aportes de los grupos originarios, heterogéneos y opuestos entre sí. Además, la conquista no termina con una destrucción total de las culturas indígenas, como — según lo advierte Roland Bernhard (2013: 160ss.) - , ocurre para muchos libros de texto alemanes y austriacos, sino con "una transformación muy profunda producida por la colonización española" (H2: 13).

\section{De "los primeros mestizos mexicanos" a la identidad hegemónica}

La transformación cultural de los primeros mestizos a la identidad hegemónica a partir del "descubrimiento" y la conquista es un tema central en los libros de texto mexicanos. El "encuentro de dos mundos" (León-Portilla, 1992) no es contextualizado en primer lugar como el final total de un episodio histórico; por el contrario, los libros narran el inicio de la historia de una fusión cultural que sugiere una identidad híbrida mexicana, que persiste hasta el día de hoy. La conquista es el escenario del nacimiento de una identidad nacional y, en sus propios términos, mestiza. ${ }^{5}$ Cómo y con qué implicaciones construyen los libros de texto esta identidad a través de la narración histórica será el objeto de las siguientes páginas.

\footnotetext{
${ }^{5}$ Para una visión de conjunto del término del mestizaje véase Schumm 1994; para un análisis específico del concepto en México, Alonso 2004
} 
Los capítulos analizados no dejan escapar ninguna oportunidad para mencionar la mezcla cultural y biológica durante la ruta de Cortés hacia Tenochtitlán. En la narración mexicana, los conquistadores encontraron en su camino al español Gonzalo Guerrero que había encallado en la costa de Yucatán años atrás y que "había adoptado las costumbres de su nueva tierra" y "procreado hijos con una princesa maya": así nacieron "los primeros mestizos mexicanos" (PR4: 53). Sin embargo, el ejemplo principal para la mezcla tanto cultural como sexual es la relación entre la Malinche y Hernán Cortés. Lo que ya comienza a verse en la narración histórica de la conquista está retomado en los siguientes capítulos bajo el título "mestizaje" (HU) o "mestizaje cultural" (H2); en ellos, la idea del mestizaje se explica refiriéndose a elementos culturales y biológicos, y se construye así una amalgama inseparable. Por ejemplo, un libro de texto de secundaria define al mestizaje de esta manera:

\footnotetext{
Mestizaje: Es la mezcla biológica y cultural de dos o más razas humanas o grupos étnicos. El término raza se refiere a las variaciones biológicas que presenta la especie humana y se muestran en características (físicas) visibles como el color de la piel, la forma y tono de los ojos y el cabello, entre otros. También es el proceso y resultado de la fusión de culturas (HU: 53).
}

Si bien en muchos casos en este proceso de mestizaje sólo se menciona a los españoles y a los indígenas (mexicas), a veces también se nombra a los "africanos": "En realidad todos participaron en un gran proceso de mezcla cultural que llamamos mestizaje. Los negros que hacían la señal de la cruz y hablaban español; [...] los indios que usaban calzón largo para cubrir sus piernas y comían carne de cerdo; todos ellos participaron en el gran proceso del mestizaje". Las implicaciones acerca del contacto intercultural son obvias: "El mestizaje implica que una cultura no suprime o desplaza a la otra, sino que se mezcla con ella" (H2: 56). Sin embargo, esta historia de la fusión cultural - la narrativa del mestizaje presentada en los libros de texto mexicanos - tiene implicaciones más profundas que se analizarán a continuación.

El concepto del mestizaje sirve para la construcción actual de una identidad hegemónica que pretende incluir diferentes culturas. Es importante notar que este tipo de inclusión cultural presentado no describe una mezcla creativa, de la cual una nueva cultura surge incontrolablemente; más bien, la narrativa del mestizaje fija las raíces culturales que alimentan a la nueva identidad hegemónica. En vez de describir un proceso abierto e involuntario del cruce cultural, los libros de texto mexicanos se enfocan en la presentación de una identidad estática que se nutre de esencias culturales claramente distinguibles. Así, cada cultura parecería haber aportado lo que se considera específico para ella, sea el cristianismo, las papas, el maíz o el cerdo. En el término "cultura" se entrelazan lo cultural y lo racial, lo que prescribe una forma de ser específica. El concepto de identidad mestiza propagado en los libros de 
texto se basa en un entendimiento de cultura como algo esencialista y total, y excluye la posibilidad de que las raíces mismas que nutren el mestizaje sean transculturales y hayan sido originadas en otras interacciones culturales.

Si el concepto del mestizaje en los libros de texto promulga una unidad en la diversidad, ¿Cómo son las relaciones de poder entre las diferentes culturas que forman parte de la identidad mestiza? Un libro de texto señala que "por supuesto, no todas las culturas involucradas compiten en las mismas condiciones: la del grupo dominante impone ciertas reglas" ( $\mathrm{H} 2:$ 56). Sin embargo, casi ninguno de los capítulos mexicanos analizados reconoce las relaciones de poder en el "encuentro de dos mundos", sino solamente las "contribuciones" (H2: 57) de cada cultura involucrada. De esta manera, los libros están orientados a comprobar que todos hayan participado y aportado lo mismo, y aunque papas y maíz, por un lado, y el cristianismo y el idioma castellano, por el otro, se encuentran en dos niveles muy distintos, se sugiere su igualdad. Así, el mestizaje se vuelve un concepto que supuestamente puede integrar lo otro en lo propio, lo que es demostrado con el ejemplo de la integración armónica de la población afrodescendiente: "En Hispanoamérica, el mestizaje favoreció su integración a la sociedad y la incorporación de algunas expresiones lingüísticas, religiosas y artísticas africanas a la cultura popular", a diferencia de las colonias francesas e inglesas donde "la segregación propició el establecimiento de comunidades afroamericanas que crearon una fuerte identidad colectiva" (HU: 53). La segregación es, en los libros de texto mexicanos, el concepto que falla allí donde el mestizaje funciona bien: en el ámbito de la integración intercultural.

El término mestizaje en el material analizado no sólo se refiere a un proceso histórico a medida que describe las transformaciones en la Nueva España, sino que es a la vez diagnóstico e interpelación identitaria para la sociedad mexicana actual. Cuanto más relevante se considera para la actualidad, tanto más armónica se vuelve la representación de las relaciones de poder inherente en el concepto de mestizaje. Por ejemplo, la identidad mestiza - producto de una mezcla cultural y biológica - muchas veces es representada metafóricamente como "comida típica":

\footnotetext{
Basta con pensar en una comida "típica mexicana" de la actualidad para entender que es resultado de la suma de contribuciones de diferentes culturas. Pensemos en un menú con sopa de pasta, tacos de pollo con frijoles y salsa verde, mango y plátano. Este menú requiere la suma de productos indígenas [...], productos europeos [...] y productos asiáticos (H2: 57).
}

La narrativa de la fusión cultural armónica culmina en la comida típica mexicana de la actualidad, ejemplo que presenta la historia propia con los cinco sentidos y que a la vez se 
esmera en que cada ingrediente de la receta cultural sea visible y distinguible. No debería extrañarnos que como referencia para la cultura actual sólo estén mencionados elementos de la cultura popular que no tienen potencial conflictivo: el pollo español y el maíz indígena ni se excluyen ni se pueden jerarquizar. Si bien con el ejemplo de la comida nacional se celebra la diferencia, unificándola en un plato, llama la atención que los libros de historia escolares sólo recurran a lo pacífico y cotidiano para tematizar la integración cultural, compatibilizando así las culturas, pero ignorando lo político en general y los conflictos culturales que se manifiestan entre políticas indígenas y el Estado en particular. Como ya bien analizaron Pacheco y otros, los libros de texto mexicanos están escritos desde una perspectiva epistémica que se concentra de manera dominante en la población mayoritaria (Pacheco et al., 2011: 528). Las culturas indígenas están a la vez presentes y ausentes: son visibles a través de sus "aportes" a la cultura mayoritaria mestiza, pero invisibles cuando se trata de sus propios conceptos de valores, prácticas e intereses políticos, que pueden ser opuestos a los supuestos hegemónicos. El discurso del mestizaje, por consiguiente, es una herramienta para "blanquear" todas las prácticas indígenas, menos las más inofensivas y consideradas banales (Kraidy, 2005: 67). Una invisibilización y un blanqueamiento aún más fuerte se dan en el caso de la cultura afroamericana.

\section{Comparar historias: dominación, hibridación y silencios epistémicos}

Desde una perspectiva de análisis del discurso y con una mirada comparativa, el acontecimiento de la conquista de América se transforma analíticamente de un hecho objetivo a un lugar discursivo con una "lógica dinámica, en el marco de la cual se negocia de manera conflictiva en el espacio discursivo sobre la ocupación particular del lugar vacío de lo general" (Nonhoff, 2007: 181). Esta ocupación discursiva funge a través del establecimiento de significados relativamente hegemónicos conectados al acontecimiento de la conquista. El análisis de la narración alemana y mexicana demuestra que la diferencia central en la representación y funcionalización de la conquista está en la manera de integrar el acontecimiento en macronarrativas más abstractas, que nutren un imaginario o una identidad de las respectivas sociedades contemporáneas. Para Hayden White, el ejemplo de la representación de la conquista en los libros de texto subraya que "a historical event is one that can truthfully be described in such a way as to serve as an element of a narrative" (White, 2008: 21). Sólo a través de su incorporación en estas narrativas se construye el evento, se define el contexto del cual surge y las consecuencias que ha tenido. Aunque con los casos alemán y mexicano se hayan tomado en cuenta dos países situados asimétricamente en el sistema 
colonial - uno era una potencia colonial; el otro, una colonia-, los libros de texto de ambos países atribuyen un significado muy importante al acontecimiento del "descubrimiento" y la conquista de América. En los libros de ambos países, la conquista de América es narrativizada como una doble frontera: el cruce de espacios culturales y temporales. Las transiciones de un espacio cultural al otro o de un tiempo al otro están, sin embargo, ocupadas discursivamente de modos muy distintos, lo que lleva a que la conquista en el presente siga siendo un evento reñido y, por lo tanto, fundamental. La comparación de los dos ruedos discursivos analizados y sus respectivos imaginarios de esta transición cultural-temporal muestra el evento histórico de la conquista en su carácter genuinamente contingente y político, producto dependiente de la construcción social en el presente. Tanto en el caso mexicano como en el alemán, con la conquista comienza la historia del presente. En este sentido, pueden condensarse dos narrativas con orientación al presente en las cuales la conquista está integrada en los libros de texto: un proceso de dominación europea en el caso alemán y un proceso de hibridación identitaria en el caso mexicano.

En los libros de texto alemanes se encuentran dos mundos anteriormente separados que equivalen a entidades culturales homogéneas y de cuyo choque resulta una diferencia clara de poder: la cultura europea domina a la otra y la reemplaza; este proceso confluye en la así llamada "europeización" del mundo. Con el "descubrimiento" y la conquista también se cumple una transición temporal que Europa, según estos medios educativos, había comenzado intrínsecamente antes y que se realiza con el viaje de Colón, la cual no es solamente espacial, sino un viaje de la Edad Media hacia la modernidad. Sin embargo, las culturas americanas, entendidas como "lo otro", están excluidas de este nuevo tiempo; Europa como entidad cultural es el portador de la modernidad - aunque con una valoración crítica- y su contraparte cultural sólo es parte del nuevo tiempo porque está europeizado. Los "indios" que sobreviven son presentados como si pertenecieran a otro tiempo. Los libros de texto alemanes enseñan que con la conquista el mundo se volvió dicotómico, con una dominación evidente de la parte europea. Por consiguiente, también las propuestas didácticas en dicho material intentan comprender el mundo moderno a partir de esta dicotomía.

En los libros de texto mexicanos se presentan tres mundos que pronto ya no serán mundos separados: Europa y los habitantes de América entran en contacto, y luego también los esclavos de África; sin embargo, la frontera entre estos tres espacios culturales no se tematiza a través de una dicotomía fija, como sucede en el caso alemán, sino que más bien se crea un nuevo espacio cultural híbrido en el cual las culturas conviven y no se reemplazan. La conquista es el nacimiento de un nuevo espacio que resulta de los tres "pasados", pero con una jerarquía obvia entre los españoles, indígenas y africanos. Esta transición de fronteras culturales se corresponde con una transición temporal, ya que los tiempos anteriormente separados y paralelos de los españoles y los aztecas se juntan para configurar un tiempo 
común que la narración mexicana considera el inicio de la historia del presente. Con el nacimiento del tiempo mexicano - metafóricamente hablando - se mueren los padres aztecas, debido a que su concepto del tiempo cíclico ya no forma parte del nuevo tiempo; sin embargo, los medios educativos mexicanos continuamente recuerdan el pasado glorioso de su "prehistoria", al trazar las tradiciones de cultura popular hasta el día de hoy. De este modo, se incluyen en la identidad hegemónica mestiza los tiempos y culturas anteriores a la conquista, pero se excluye a la vez una continuidad de las respectivas entidades culturales y sus concepciones temporales específicas. Así, una simultaneidad de concepciones culturales y temporales heterogéneas es negada.

Finalmente, en las versiones alemana y mexicana de la historia en los libros escolares aquí estudiados se observan dos maneras de tematizar el inicio del colonialismo, las cuales fungen como el conocimiento hegemónico en sus respectivos ruedos discursivos. En el caso mexicano, el colonialismo genera un proceso de hibridación sobre el cual un país colonizado crea una identidad nacional fuerte. Los libros de texto mexicanos están escritos desde una perspectiva subalterna -en comparación con la posición europea-, que trata de salir del lugar marginal originado por el colonialismo y establecer una identidad nacional que no es presentada como víctima de la colonización, sino como una apropiación positiva de ella. Por el contrario, los libros de texto alemanes son fruto de una perspectiva hegemónica. Del colonialismo resulta una asimetría cultural que los libros tratan de entender, pero que de todas maneras reproducen en y para el presente. En este contexto se establece la particularidad europea como una norma para lo global, ya que se universaliza la "historia local" como "diseño global" (Mignolo, 2003). La historia y actualidad global, según los libros alemanes, es sólo entendida en relación con lo europeo.

A pesar de que pueden contrastarse las dos representaciones y funcionalizaciones históricas en términos de hegemonía y subalternidad, no debe perderse de la vista que los dos casos comparten bases epistémicas y mecanismos de exclusión semejantes. Si bien la historiografía presenta el pasado a través de selección y semantización, este análisis además demuestra que en todos los libros de texto analizados lo conflictivo del presente está desterrado hacia el pasado y que las continuidades están suprimidas y narrativizadas como discontinuidades; son las culturas indígenas y afrodescendientes que supuestamente - de una u otra manera - no han transitado la doble frontera de lo espacial-cultural y lo temporal, por lo cual quedan excluidas de la cultura contemporánea. Los libros de texto mexicanos reconocen que lo indígena es una raíz importante de la identidad mexicana, pero debido a su proyección idealizada en un "pasado glorioso" o el interés en la cultura popular desconocen la transcendental presencia política y cultural indígena en el México contemporáneo (Pacheco et al., 2011: 534). En este sentido, dentro del ruedo discursivo mexicano la representación del mestizaje no parece ser subalterna, sino que se consolida como un mecanismo 
hegemónico de exclusión. Por otro lado, la representación de la conquista en los libros de texto alemanes excluye un continente entero como actor de la historia universal y describe una cultura azteca pasada cuyas características y aportes los alumnos deben comprender, pero sin contar con la posibilidad de romper con la epistemología eurocéntrica que forma la base de la narración.

\section{A modo de conclusión}

El propósito de este artículo fue analizar con una mirada comparativa la narrativización y funcionalización del evento histórico de la conquista de América en los libros de texto actuales de Alemania y México. Con una heurística del análisis del discurso y el análisis narrativo, basado en las teorías historiográficas y decoloniales, se llegó a la conclusión de que en los dos ruedos discursivos la conquista es integrada en macronarrativas distintas, que resultan en diferentes imaginarios de (inter)culturalidad para las sociedades contemporáneas: el mestizaje para México y la dominación europea para Alemania. Sin embargo, desde la perspectiva de las teorías decoloniales, los libros de texto tanto alemanes como mexicanos no sólo son "representaciones del mundo colonial" — para retomar la expresión de Macgilchrist y Otto (2014: 4) - sino debido a su epistemología también son representaciones coloniales del mundo colonial, ya que a través de su historiografía y sus implicaciones perpetúan la diferencia colonial, aunque el sistema de dominación del colonialismo haya desaparecido. En distintos niveles, ambas narraciones históricas escolares coinciden en la producción de silencios epistémicos que excluyen de una u otra manera lo indígena de la representación histórica. El estudio aquí presentado hace visible un mecanismo de exclusión epistémico que el sociólogo portugués Boaventura de Sousa Santos denomina "la producción de ausencias" (Santos, 2009: 110). Al excluir las culturas indígenas del presente y desplazarlas al pasado, el presente queda reducido a sus expresiones hegemónicas a través de la historia. Ninguno de los libros de texto aquí analizados logra reflejar esta consecuencia de la historiografía: "el silencio en la historia mundial" y "la extinción y banalización" de otros presentes que constituyen otras historias (Trouillot, 2002: 102ss.); por consiguiente, en un plano epistemológico decolonial hay que subrayar que los medios educativos de ambos países consolidan la exclusión de perspectivas indígenas y, al mismo tiempo, legitiman este silenciamiento al no tematizar la contingencia propia de su representación. Los libros de texto, por medios narrativos y didácticos hacen callar continuamente a Cem Anahuac, el mundo, según la denominación que los aztecas le dieran (Dussel, 1993: 108). 


\section{Fuentes}

Libros de texto consultados (con abreviaturas)

Baumgärtner, Ulrich (coord.) (2014), Horizonte 2. Geschichte für Gymnasien in Hessen und im Saarland ( $\mathrm{HZ})$, Westermann, Braunschweig.

Berger-v. d. Heide, Thomas y Oomen, Hans-Gert (coords.) (2013), Entdecken und Verstehen. Vom Mittelalter bis zur Entstehung der Vereinigten Staaten. Schülerbuch Real- und Gesamtschule Hessen (Ev), Cornelsen, Berlín.

Christoffer, Sven et al. (coords.) (2012), Zeitreise 2. Schülerbuch Haupt-, Real und Gesamtschule Hessen (ZR), Klett, Stuttgart-Leipzig.

Escalante Gonzalbo, Pablo et al. (2011), Historia 2. Secundaria. Conect@ Entornos (H2), sM de Ediciones, México.

Laschewski-Müller, Karin y Rauh Robert (coords.) (2010), Kursbuch Geschichte. Von der Antike bis zur Gegenwart. Neue Ausgabe Hessen (KC), Cornelsen, Berlín.

Sánchez Cervantes, Alberto et al. (2012), Historia Universal. Secundaria (HU), Ríos de Tinta, México.

Secretaría de Educación Pública (ed.) (2009), Historia. Sexto Grado (PR6), SEP, México. (2010), Historia. Cuarto Grado (PR4), SEP, México.

Literatura cientifica

Alonso, Ana María (2004), "Conforming Disconformity: 'Mestizaje', Hybridity, and the Aesthetics of Mexican Nationalism", Cultural Anthropology, vol. 19, núm. 4, pp. 459-490.

Anzures, Tonatiuh (2011), "El libro de texto gratuito en la actualidad. Logros y retos de un proyecto cincuentenario", Revista Mexicana de Investigación Educativa, vol. 16, núm. 49, pp. 363-388.

Bernhard, Roland (2013), Geschichtsmythen über Hispanoamerika. Entdeckung, Eroberung und Kolonisierung in deutschen und österreichischen Schulbüchern des 21. Jahrhunderts, V\&R Unipress, Göttingen.

Böttcher, Nikolaus (2013), Kontinuität und Brüche in Hispanoamerika, vs, Wiesbaden.

Certeau, Michel de (1991), Das Schreiben der Geschichte, Campus, Frankfurt-Nueva York.

Chakrabarty, Dipesh (1992), "Postcoloniality and the Artifice of History: Who Speaks for "Indian" Pasts?", Representations, núm. 37, pp. 1-26.

Choppin, Alain (2001), "Pasado y presente de los manuales escolares", Revista Educación y Pedagogía, vol. 13, núm. 29-30, pp. 207-229.

Díaz Barriga, Ángel (2006), "El enfoque de competencias en la educación. ¿Una alternativa o un disfraz de cambio?", Perfiles Educativos, vol. 28, núm. 111, pp. 7-36.

Dussel, Enrique (1993), Von der Erfindung Amerikas zur Entdeckung des Anderen. Ein Projekt der Transmoderne, Patmos, Düsseldorf.

Eckert, Andreas y Albert Wirz (2002), "Wir nicht, die anderen auch: Deutschland und der Kolonialismus", en Sebastian Conrad y Shalini Randeria (coords.), Jenseits des Eurozentrismus. Postkoloniale Perspektiven in den Geschichts- und Kulturwissenschaften, Campus, Frankfurt-Nueva York, pp. 372-392.

Escobar, Arturo (2003), "'Mundos y conocimientos de otro modo'. El programa de investigación de modernidad/colonialidad latinoamericano", Tabula Rasa, núm. 1, pp. 51-86.

Heinze, Karsten (2014), "On the Pedagogization of Knowledge Orders - Discourse-Analytical Approaches and Innovation-Theoretical Perspectives", en Petr Knecht et al. (coords.), Methodologie und Methoden der Schulbuch- und Lehrmittelforschung, Klinkhardt, Bad Heilbrunn, pp. 74-84. 
Hessisches Kultusministerium (2011), "Bildungsstandards und Inhaltsfelder: Das neue Kerncurriculum für Hessen; Sekundarstufe I - Gymnasium; Geschichte: 11", documento pdf, disponible en: <curricula-depot.gei.de/bitstream/handle/11163/805/720535174_2011_A.pdf? sequence=2> (fecha de consulta: 10/02/2015).

Hobsbawm, Eric (coord.) (1984), The Invention of Tradition, Cambridge University Press, Cambridge.

Höhne, Thomas (2003), Schulbuchwissen. Umrisse einer Wissens- und Medientheorie des Schulbuchs, Johann-Wolfgang-Goethe-Universität, Frankfurt-Main.

Keller, Reiner (2010), "Kompetenz-Bildung: Programm und Zumutung individualisierter Bildungspraxis. Über Möglichkeiten einer erweiterten Bildungssoziologie”, en Thomas Kurtz y Michaela Pfadenhauer (coords.), Soziologie der Kompetenz, vs, Wiesbaden, pp. 29-48.

Knecht, Petr et al. (coords.) (2014), Methodologie und Methoden der Schulbuch- und Lehrmittelforschung/ Methodology and methods of research on textbooks and educational media, Klinkhardt, Bad Heilbrunn.

Kraidy, Marwan M. (2005), Hybridity, or the Cultural Logic of Globalisation, Temple University Press, Philadelphia.

Lamoneda Huerta, Mireya (2004), "La enseñanza de 'El Descubrimiento y la conquista de América', en los libros de texto", en Carmen Castañeda García et al. (coords.), Lecturas y lectores en la historia de México, CIESAS-El Colegio de Michoacán-Universidad Autónoma del Estado de Morelos, México, pp. 177-192.

León-Portilla, Miguel (1992), "Encuentro de dos mundos", Estudios de Cultura Náhuatl, núm. 22, pp. $15-27$.

Macgilchrist, Felicitas y Otto, Marcus (2014), "Schulbücher für den Geschichtsunterricht, Version: 1.0", Docupedia-Zeitgeschichte, documento pdf, disponible en: <docupedia.de/zg/Schulbuecher> (fecha de consulta: 15/02/2015).

Mignolo, Walter D. (2003), Historias locales/diseños globales. Colonialidad, conocimientos subalternos y pensamiento fronterizo, Akal, Madrid.

Neyra Galicia, Alma Rosa (2010), "El bachillerato mexicano y la política educativa: desde sus inicios hasta la educación basada en competencias", Textual. Análisis del medio rural latinoamericano, núm. 55, pp. 63-82.

Nonhoff, Martin (2007), "Politische Diskursanalyse als Hegemonieanalyse", en Martin Nonhoff (coord.), Diskurs - radikale Demokratie - Hegemonie. Zum politischen Denken von Ernesto Laclau und Chantal Mouffe, transcript, Bielefeld, pp. 173-193.

Osterhammel, Jürgen y Jansen, Jan C. (2012), Kolonialismus: Geschichte, Formen, Folgen, Beck, Múnich.

Pacheco, Lourdes C. et al. (2011), "Los pueblos indios en los libros de texto gratuitos", Revista Mexicana de Investigación Educativo, vol. 16, núm. 49, pp. 525-544.

Pérez Siller, Javier (coord.) (1992), La "découverte" de l'Amérique? Les regards sur l'autre à travers les manuels scolaires du monde, L'Harmattan, París.

Pieters, Jürgen (2000), "New Historicism: Postmodern Historiography between Narrativism and Heterology", History and Theory, vol. 39, núm. 1, pp. 21-38.

Santos, Boaventura de Sousa (2009), Una epistemología del sur: La reinvención del conocimiento y la emancipación social, Siglo XXI-CLACSO, México.

Schumm, Petra (1994), "Mestizaje und culturas híbridas - kulturtheoretische Konzepte im Vergleich", en Birgit Scharlau (coord.), Lateinamerika denken. Kulturtheoretische Grenzgänge zwischen Moderne und Postmoderne, Gunter Narr Verlag, Tübingen, pp. 59-80.

Secretaría de Educación Pública (2011), "Plan de Estudios 2011. Educación Básica: 35", documento pdf, disponible en: <curricula-depot.gei.de/bitstream/handle/11163/2306/796516677_2011_A. pdf? sequence $=2>$ (fecha de consulta: 10/02/2015). 
Subsecretaría de Educación Media Superior (2013), "Historia de México 1. Serie Programas de Estudio: 30", documento pdf, disponible en: <curricula-depot.gei.de/bitstream/handle/11163/229 0/796648050_2013_A.pdf? sequence=2> (fecha de consulta: 10/02/2015).

Stöber, Georg (2010), "Schulbuchzulassung in Deutschland. Grundlagen, Verfahrensweisen und Diskussionen", Eckert.Beiträge 2010/3, pp. 1-24.

Todorov, Tzvetan (1998), La conquista de América. El problema del otro, Siglo XXI, México.

Trouillot, Michel-Rolph (2002), "Undenkbare Geschichte. Zur Bagatellisierung der haitischen Revolution", en Sebastian Conrad y Shalini Randeria (coords.), Jenseits des Eurozentrismus. Postkoloniale Perspektiven in den Geschichts- und Kulturwissenschaften, Campus, Frankfurt-Nueva York, pp. 84-115. Viehöver, Willy (2006), "Diskurse als Narrationen", en Reiner Keller (coord.), Handbuch sozialwissenschaftliche Diskursanalyse. Tomo I: Theorien und Methoden, vs, Wiesbaden, pp. 179-208.

White, Hayden (2008), "The Historical Event", Differences, vol. 19, núm. 2, pp. 9-34.

Daniel Schumann. Estudiante de maestría en sociología de la Albert-Ludwigs Universität Freiburg, Alemania. B. A. en sociología y antropología cultural. Líneas de investigación: sociología del conocimiento, sociología cultural, descolonialidad y estudios postcoloniales. Se encuentra en proceso de publicación: Daniel Schumann (2016), "Koloniale Wege in die moderne Welt. Zur Vergegenwärtigung der Eroberung Amerikas in aktuellen deutschen und mexikanischen Geschichtsschulbüchern”, Eckert.Beiträge 2016.

Recibido: 15 de abril de 2016

Aceptado: 2 de mayo de 2016 